\title{
Química de la alimentación vegana relacionada con la salud mental
}

Chemistry of vegan alimentation related to mental health

\author{
Susana Martínez-Casamayor ${ }^{1}$, Pedro Andreo-Martínez ${ }^{2 *}$
}

Citación/Citation: Martínez-Casamayor S, AndreoMartínez P. Rev Dis Cli Neuro. 2021; (8)1: 52-59.

Available from: https://doi.org/10.14198/DCN.19780

Recibido/Received: 21/04/2021

Aceptado/Accepted: 21/04/2021

Financiación/Funding: Este estudio no ha recibido financiación.

Conflicto de intereses/Conflicts of Interest: Los autores declaran no tener conflicto de intereses.

\section{(ㄷ) 2021 Susana Martínez-Casamayor y Pedro} Andreo-Martínez

Licencia/Licence: This work is licensed under a Creative Commons Attribution 4.0 International License (CC BY 4.0).

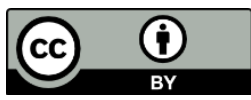

Editorial: Universidad de Alicante (España) Autores/Authors:

${ }^{1}$ Boticarea. Murcia. España, https://orcid.org/00000003-4702-6593

2 Departamento de Química Agrícola, Geología y Edafología. Universidad de Murcia. España, https://orcid.org/0000-0001-6535-5492

* Contact for correspondence: pam11@um.es
Resumen: La popularidad de una dieta basada en productos vegetales, tradición inveterada en las culturas orientales, está proliferando en el mundo occidental. El veganismo es un destacado movimiento social del siglo XXI al que se le atribuyen multitud de beneficios relacionados con el bienestar físico entre los que se reconocen un menor riesgo de obesidad, diabetes, problemas cardiovasculares (altos niveles de colesterol y presión arterial), e incluso el de padecer algunos tipos de cáncer. Sin embargo, son escasos los estudios que relacionan esta dieta con los posibles efectos adversos que puede tener en la salud mental. "The Vegan Society" enumera el calcio, los ácidos grasos esenciales, el yodo, el hierro, las proteínas, y las vitaminas D y B12 como los nutrientes esenciales que pueden ser de preocupación al seguir una dieta vegana. Es por ello que los veganos pueden presentar, en algunos casos, carencias nutricionales relacionadas en la actualidad con una salud mental. La transición a esta dieta ha de llevarse a cabo contando con suficiente información para permitir alcanzar un equilibrio nutricional adecuado, que puede estar mermada o sensacionalizada en los medios de comunicación más empleados en la actualidad. También debe ser considerada la emergente psiquiatría nutricional para estudiar, de manera científica, la influencia de la dieta en las enfermedades mentales, tanto a nivel preventivo como para su tratamiento, las cuales son la principal causa de discapacidad en el mundo. Es por ello que los trastornos mentales y de comportamiento, que están en aumento en nuestra sociedad, pueden estar relacionados con los déficits nutricionales contribuyendo a la aparición y/o mantenimiento de muchos de ellos debido a una nutrición deficitaria y/o a un desequilibrio químico. Por consiguiente, futuros estudios deberían tener presente, entre otras consideraciones, la mencionada psiquiatría nutricional a largo plazo para concluir con información científica rigurosa sus resultados.

Palabras clave: Dieta; Enfermedades mentales; Nutrientes; Salud mental; Veganismo. 


\begin{abstract}
The popularity of a diet based on plant products, an inveterate tradition in Eastern cultures, is proliferating in the Western world. Veganism is a prominent social movement of the 21st century to which a multitude of benefits related to physical well-being are attributed, among which are recognized a lower risk of obesity, diabetes, cardiovascular problems (high levels of cholesterol and blood pressure), and even the of suffering from some types of cancer. However, there are few studies that link this diet with the possible adverse effects it can have on mental health. The Vegan Society lists calcium, essential fatty acids, iodine, iron, protein, and both D and B12 vitamins as essential nutrients that may be of concern when following a vegan diet. That is why vegans can present, in some cases, nutritional deficiencies currently related to mental health. The transition to this diet must be carried out with enough information to allow an adequate nutritional balance to be achieved, which may be diminished or sensationalized in the most used media today. The emerging nutritional psychiatry should also be considered to study, in a scientific way, the influence of diet on mental illnesses, both preventively and for their treatment, which are the main cause of disability in the world. That is why mental and behavioral disorders, which are on the rise in our society, may be related to nutritional deficits, contributing to the appearance and / or maintenance of many of them due to poor nutrition and / or a chemical imbalance. Therefore, future studies should bear in mind, among other considerations, the aforementioned nutritional psychiatry in the long term to conclude their results with rigorous scientific information.
\end{abstract}

Keywords: Diet; Mental illness; Mental health; Nutrients; Veganism.

\title{
1. Introducción
}

El tipo de dieta vegetariana es un término general que incluye a veganos, lactovegetarianos, ovovegetarianos y ovolactovegetarianos como patrones dietéticos principales. Una dieta vegana se caracteriza por el rechazo de todos los productos de origen animal, como carnes, pescados, mariscos, productos lácteos, huevos y miel. Los lactovegetarianos incorporan a la dieta vegana productos lácteos, los ovovegetarianos incorporan solo huevos y los ovolactovegetarianos incluyen tanto lácteos como huevos. Mientras que un patrón dietético sin ninguna restricción se conoce como un tipo de dieta omnívora [1].

El vegetarianismo, que se remonta a la antigua Grecia asociado a Pitágoras y sus seguidores, comienza una transición al veganismo cuando miembros de "The Vegetarian Society" londinense argumentaron sobre los daños que sufrían los animales como consecuencia de la producción láctea y la cría de huevos. La búsqueda de un término más estricto que promueva una dieta basada en la abstención de los productos de origen animal y, a su vez, un estilo de vida basado en la ética, dio lugar al veganismo en la década de los 40 de la mano de Donald Watson quien acuño en 1944 el término "vegan", el cual fue uno de los fundadores de una nueva sociedad que se conoce como "The Vegan Society". En la actualidad se define vegano como un vegetariano estricto que no consume alimentos de origen animal ni productos lácteos más allá de la exclusión dietética, ya que el adoptar esta dieta puede deberse a multitud de motivos donde la salud o las razones éticas como la defensa de los animales o la protección del medio ambiente son los 
principales. "The American Vegan Society" declara que los veganos viven de los productos del reino vegetal ya que el veganismo es compasión en acción. Es una filosofía, una dieta y un estilo de vida, que ha tomado gran protagonismo en los últimos años y hay cada vez más personas que la eligen [2].

Por otra parte, en los primeros períodos de la civilización griega, la locura se consideraba un castigo divino por transgresiones mayores o menores. Los dioses enviaban espíritus malignos, personificados en las diosas Lisa y Manía para poseer al enfermo. El tratamiento consistía en ritos expiatorios de los sacerdotes, mediando en las oraciones del enfermo, en templos dedicados al dios de la medicina, Asclepios. Los procedimientos incluían dieta, masaje, sueño, baños y sugerencias sacerdotales. En esa misma época, comienza a gestarse lo que más tarde daría lugar a un cambio fundamental en la forma de abordar el estudio de las psicopatologías. Algunos pensadores se alejaron de la idea sobrenatural y mística que relacionaba las enfermedades mentales con la demonología y la brujería. Pitágoras destacó la importancia de identificar los principios científicos que explicaran el comportamiento humano, afirmando que el cerebro era el órgano del intelecto y fuente de perturbaciones mentales. Burton (1576-1640) escribió “La Anatomía de la Melancolía", considerado como el primer tratado sobre la depresión, donde identifica la culpa, el deterioro corporal y las dietas inadecuadas entre las causas de la melancolía [3].

La Organización Mundial de la Salud (OMS) informó en 2017 que las enfermedades mentales eran la principal causa de discapacidad en todo el mundo, ejerciendo también influencia en las enfermedades cardiovasculares que son la principal causa de mortalidad a nivel mundial. La OMS define la salud mental como un estado de bienestar en el que cada individuo se da cuenta de su propio potencial, puede hacer frente a las tensiones normales de la vida, puede trabajar de forma productiva y fructífera, y puede hacer una contribución a su comunidad [4]. La dieta vegetariana, a menudo, se ha asociado con una salud mental reducida en investigaciones anteriores [5].

\section{Objetivo}

La finalidad de este trabajo es transmitir información actual sobre la relación que puede darse entre los hábitos alimentarios restrictivos asociados a la dieta vegana y el papel que pueden desempañar en las enfermedades mentales.

\section{Discusión}

\subsection{Dieta vegana y posibles déficits nutricionales}

Existen múltiples beneficios para la salud que pueden motivar a seguir una dieta vegana, pero también pueden darse deficiencias que pueden ocasionar resultados adversos. Diversos estudios apuntan a que este tipo de dieta puede influir favorablemente en los niveles de colesterol, lípidos, presión arterial y peso, y reduce el riesgo de enfermedades como la obesidad, la diabetes, problemas cardiovasculares o algunos tipos de cáncer. Sin embargo, a pesar de estos beneficios, “The Vegan Society" detalla que hay ciertos nutrientes que pueden ser de preocupación al practicar una dieta vegana, de acuerdo con los hallazgos de la literatura científica, y muestra cómo ciertas deficiencias pueden tener consecuencias graves para la salud. Además, proporciona explicaciones detalladas de por qué los veganos pueden correr un mayor 
riesgo de ciertas deficiencias nutricionales de calcio, ácidos grasos esenciales, yodo, hierro, proteínas, vitamina D y vitamina B12 [2].

Los alimentos de origen vegetal son fuente de proteínas incompletas, aportan ciertos aminoácidos, y solo son digeribles en un $85 \%$. Los alimentos proteicos completos son de origen animal y tienen todos los aminoácidos esenciales, los que el cuerpo no puede producir por sí mismo [2]. Las proteínas están incrustadas en la bicapa lipídica de las membranas neuronales y la conformación o estructura cuaternaria de estas proteínas es sensible a los componentes lipídicos. Tienen funciones celulares críticas, ya que actúan como transportadores y receptores [6], ejerciendo un rol importante en la bioquímica del cerebro.

Un estudio concluyó que la ingesta diaria de vitamina B12 en la dieta vegana estaba muy por debajo de los niveles recomendados, ya que es un nutriente de origen animal y tanto vegetarianos como veganos no ingieren [2]. La deficiencia de vitamina B12 puede manifestarse con signos hematológicos, gastrointestinales y neuropsiquiátricos como depresión, demencia, catatonia y delirio. Los signos y síntomas neuropsiquiátricos secundarios a la deficiencia de vitamina B12 pueden explicarse por la participación de la vitamina B12, así como del folato y la homocisteína, en el metabolismo de transferencia de carbono (metilación) necesario para la producción de serotonina. Los estudios han demostrado un alto riesgo de depresión en las mujeres mayores con deficiencia de vitamina B12 en comparación con las mujeres sanas [7, 8]. Sirva, a modo de ejemplo, un caso clínico de delirio por deficiencia de vitamina B12 atribuido a la alimentación vegetariana. La paciente tenía antecedentes de un episodio depresivo mayor hace 5 años. Una evaluación psicométrica mostró una puntuación de 27 en la MADRS (Montgomery Asberg Depression rating scale) y 22/30 en el MMSE (Mini-Mental State Examination). En el test del reloj obtuvo una puntuación de 20 en la DRS (Delirium Rating Scale), lo que indica delirio con presencia de síntomas depresivos graves y deficiencias cognitivas. Al no encontrar otra causa de delirio, la paciente fue tratada empíricamente con inyecciones semanales de $1000 \mu \mathrm{g}$ de cianocobalamina (vitamina B12). Cuatro semanas después del episodio, se encontraron niveles de funcionamiento premórbidos y remisión total de los síntomas depresivos, mostrando una puntuación de 4 en el MADRS [9].

La vitamina $\mathrm{D}$ es nutriente esencial que se obtiene en alimentos y se produce por el cuerpo mediante la exposición a la luz solar. Está limitada en los alimentos no fortificados de la dieta vegana y puede ser recomendable la ingesta de productos enriquecidos, sobre todo para aquellos que viven en latitudes altas, ya que son las mejores fuentes de esta vitamina para los veganos. Aproximadamente la mitad de los veganos están potencialmente en riesgo de deficiencia de vitamina D porque la mayoría no toman suplementos ni multivitamínicos [2].

Los minerales que pueden ser de preocupación en una dieta vegana son el zinc, el hierro y el calcio. El zinc es de reducida biodisponibilidad, definida como el grado y la velocidad a la que una sustancia se absorbe en un sistema vivo o se pone a disposición en el lugar de actividad fisiológica, en fuentes vegetales y existen estudios que han demostrado que los vegetarianos tienen niveles más bajos de zinc [10]. El hierro que se consume en las dietas basadas en vegetales es el hierro tipo no hemo y tiene una menor capacidad de absorción que el hierro hemo, obtenido de los alimentos de origen animal. El calcio se asocia a los productos lácteos, aunque se puede encontrar en alimentos vegetales debido a la fortificación deliberada. Es importante tener en cuenta que la biodisponibilidad de estos minerales se puede ver disminuida por la presencia de secuestradores iónicos como oxalatos, fitatos y fibras que se encuentran en las dietas veganas [2]. 
Otra de las preocupaciones de los veganos es la ingesta de cantidades inadecuadas de ácidos grasos esenciales, en concreto los ácidos grasos $\omega$-3. En este sentido, se sabe que el Homo sapiens evolucionó en un entorno rico en ácidos grasos poliinsaturados $\omega$-3 obtenidos del consumo de pescado y marisco, lo que pudo facilitar la encefalización homínida [11]. Las dietas veganas, en general, carecen de aportes significativos de ácido docosahexaenoico (DHA) y ácido eicosapentaenoico (EPA), ya que son importantes para la salud cardiovascular y las funciones cerebrales [2]. Además, las deficiencias dietéticas de estos ácidos grasos están asociadas con un mayor riesgo de desarrollar diversos trastornos psiquiátricos, como depresión, trastorno bipolar, esquizofrenia, demencia, déficit de atención e hiperactividad y autismo [11]. El DHA se asocia con la estabilidad de la membrana neuronal y con el funcionamiento de la neurotransmisión dopaminérgica y serotoninérgica, y se ha demostrado que los ácidos grasos $\omega$-3 tienen cierta actividad antiinflamatoria y que los ácidos grasos $\omega-6$ son precursores de eicosanoides proinflamatorios, tales como prostaglandinas y tromboxanos [12]. La ingesta de ácidos grasos $\omega-3$ en occidente ha descendido drásticamente en el último siglo, mientras que se ha incrementado la ingesta de ácidos grasos $\omega-6$, lo cual incrementa el ratio $\omega-6 / \omega-3$, que se traduce en un estado proinflamatorio [12]. Aunque el ácido linolénico (ALA), un ácido graso $\omega-3$, puede metabolizarse en EPA y DHA, las dietas veganas son ricas en ácidos grasos $\omega-6$ que perjudican esta biosíntesis [2]. Además, la conversión biológica es relativamente lenta e ineficaz [11] y se estima que solo del 5 al $15 \%$ del ALA se convierte finalmente en DHA [6].

El interés en los efectos terapéuticos de los ácidos grasos $\omega$-3 reside en que las membranas de los eritrocitos de las personas con esquizofrenia y depresión presentan concentraciones reducidas de este tipo de ácidos grasos. Además, diversos estudios apuntan a que existen asociaciones entre la cantidad de pescado consumido y la incidencia de los trastornos depresivos, que curiosamente han aumentado desde 1945, además de bipolaridad y homicidio $[6,11]$. Con respecto a indicadores bioquímicos, varios estudios han encontrado una correlación negativa entre el contenido de ácidos grasos $\omega$-3 en la sangre de pacientes deprimidos y una correlación negativa entre el contenido de EPA en los fosfolípidos de los glóbulos rojos con la gravedad de la depresión, mientras que se correlaciona positivamente la proporción de EPA con los síntomas clínicos de la depresión. El mecanismo mediante el cual los ácidos grasos $\omega-3$ pueden ejercer una influencia significativa en la depresión es a través de la modulación de citoquinas, que incluyen interleucina-1 beta (IL-1 $\beta$ ), -2 y -6 , interferón-gamma y factor de necrosis tumoral alfa (TNF $\alpha$ ). La modulación de estas citoquinas puede tener efectos directos e indirectos sobre el sistema nervioso central ya que favorecen la disminución de la disponibilidad de precursores de neurotransmisores, activación del eje hipotalámico-pituitario y alteraciones del metabolismo de neurotransmisores y ARNm de neurotransmisores. Los ácidos grasos $\omega-3$, y el EPA en particular, son inhibidores de citoquinas proinflamatorias como IL-1 $\beta$ y TNF $\alpha$ [6].

\subsection{Implicaciones de la alimentación vegana en la salud mental}

Una revisión sistemática sobre la incidencia del consumo de carne en la depresión, ansiedad y enfermedades relacionadas, encontró que once estudios demostraban que la abstención de la ingesta de carne se asoció con una peor salud psicológica, cuatro fueron equívocos y tres mostraron que los abstencionistas de carne mostraban mejores resultados. Los estudios de mayor calidad mostraron que aquellos individuos que evitaron el consumo de carne presentaban tasas o riesgo significativamente más altos de depresión, ansiedad y/o conductas de autolesión [4]. 
Sin embargo, en marzo de 2021 se realizó una búsqueda en la base de datos científica "Web of Science" con las palabras clave "vegan diet" AND "mental illness" y se encontraron solo cinco artículos, los cuales apuntan a que la práctica del veganismo también puede ser relacionada con efectos positivos sobre la salud mental $[1,5,13-15]$.

Uno de los artículos realizó un estudio transversal con población alemana, rusa y estadounidense, y un estudio longitudinal con estudiantes universitarios alemanes y chinos. Se utilizó la versión corta de las Escalas de Depresión, Ansiedad y Estrés (DASS-21) y se concluyó que el vegetarianismo solo está asociado con la ansiedad y la depresión en estudiantes chinos a largo plazo. Los autores de este estudio no dan una razón concreta de este hecho [5]. Sin embargo, un metaanálisis sobre la prevalencia de la depresión en estudiantes chinos concluyó que estos estudiantes están sometidos a varios estresores entre los que se incluyen las interacciones sociales ampliadas y las situaciones cambiantes tanto económicas como residenciales [16].

Dos estudios realizados en un entorno corporativo con una duración de entre 18 y 22 semanas, evaluaron el efecto de la dieta vegana en la salud mental y en la productividad laboral. Para ello, reclutaron a estadounidenses con índice de masa corporal (IMC) $>25 \mathrm{~kg} \mathrm{~m}^{-2} \mathrm{y} / \mathrm{o}$ diabetes tipo II que no siguieran una dieta vegana en la actualidad, donde destacaron participantes del sexo femenino. Al grupo de intervención se le pidió que tomara un multivitamínico para cumplir los requisitos de vitamina B12 y el bienestar emocional se evaluó mediante la Encuesta general de salud (SF-36). Ambos estudios concluyeron que una dieta vegana favorece la productividad laboral y la salud mental [13, 14].

En otro análisis transversal, una mayor restricción de la ingesta de productos de origen animal se asoció con un IMC más bajo, pero no con la depresión medida con la escala del Centro de Estudios Epidemiológicos-Depresión (CES-D). Si hubo una asociación significativa de las variables demográficas con IMC, personalidad y síntomas depresivos con diferencias significativas respecto al sexo [15].

Por último, un estudió comparó el estado de salud de corredores de resistencia veganos y omnívoros. Destacaron participantes femeninos y de nacionalidad alemana, evaluándose su salud mental mediante respuesta positiva o negativa a la pregunta “¿Estás bajo presión y / o estás sufriendo estrés?”. Los veganos obtuvieron las puntuaciones más altas, aunque no hubo asociación significativa entre el tipo de dieta y la salud mental. Estos hallazgos apoyan la idea de que adherirse a una dieta vegana es (al menos) una alternativa igual de saludable que una dieta omnívora para corredores de resistencia [1].

\section{Conclusiones}

El estudio de las implicaciones de la práctica del veganismo en la salud mental está en el foco de interés hoy en día. La literatura científica indica que las deficiencias nutricionales están asociadas a una salud mental reducida. Sin embargo, algunos estudios indican que la dieta vegana no debe suponer un riesgo y que es, incluso, beneficiosa para la salud mental. No obstante, algunos aspectos sobre estas investigaciones deben ser tomados en consideración. Por ejemplo, en los estudios realizados en el ámbito laboral de EEUU, se podrían relacionar fácilmente las emociones positivas asociadas a la práctica de la dieta vegana con otras motivaciones referentes a la satisfacción de seguir una dieta con conocidas ventajas para prevenir y tratar el sobrepeso y la diabetes que mostraban los participantes. Un cambio en la rutina diaria también 
puede ser considerado como un factor determinante para mantener una actitud mental positiva, sobre todo en el entorno laboral, por lo que los estudios deberían haber tenido en cuenta los resultados más a largo plazo, tanto por este motivo como por valorar las posibles deficiencias nutricionales. Además, puede resultar insuficiente medir la salud mental de personas que realizan deporte con un único ítem al respecto ya que la práctica de ejercicio también está relacionada con una disminución del estrés, y no solo con el tipo de dieta que se adopte.

Por lo tanto, futuros estudios también deberían tener en consideración la psicología nutricional para poder encontrar una relación real entre la dieta vegana y las enfermedades mentales ya que las principales limitaciones encontradas en los estudios son: (I) miden la salud mental con un número limitado de ítems, (II) no consideran adecuadamente los sesgos de medir la ingesta dietética mediante autoinforme, y (III) cuando concluyen que la dieta vegana favorece la salud mental no sopesan la influencia de otros motivos en sus resultados. En consecuencia, estudios longitudinales pueden ser precisos para obtener resultados objetivos respecto a la práctica del veganismo a largo plazo.

\section{Referencias}

1. Wirnitzer K, Boldt P, Lechleitner C, Wirnitzer G, Leitzmann C, Rosemann T, et al. Health Status of Female and Male Vegetarian and Vegan Endurance Runners Compared to Omnivores - Results from the NURMI Study (Step 2). Nutrients 2019, 11 (1): 29. doi: https://doi.org/10.3390/nu11010029

2. Mann S. More Than Just A Diet: An Inquiry Into Veganism. Anthropology Senior Theses. Paper 156. 2014.

3. Belloch A, Bonifacio S, Francisco R. Manual de psicopatología, Madrid, España: Macgraw-hill; 2008.

4. Dobersek U, Wy G, Adkins J, Altmeyer S, Krout K, Lavie CJ, et al. Meat and mental health: a systematic review of meat abstention and depression, anxiety, and related phenomena. Crit Rev Food Sci Nutr 2021, 61 (4): 622-635. doi: https://doi.org/10.1080/10408398.2020.1741505

5. Lavallee K, Zhang XC, Michalak J, Schneider S, Margraf J. Vegetarian diet and mental health: Crosssectional and longitudinal analyses in culturally diverse samples. J Affect Disord 2019, 248: 147-154. doi: https://doi.org/10.1016/j.jad.2019.01.035

6. Logan AC. Omega-3 fatty acids and major depression: a primer for the mental health professional. Lipids Health Dis 2004, 3: 25-25. doi: https://doi.org/10.1186/1476-511X-3-25

7. Pawlak R. Is Vitamin B12 Deficiency a Risk Factor for Cardiovascular Disease in Vegetarians? Am J Prev Med 2015, 48 (6): e11-e26. doi: https://doi.org/10.1016/j.amepre.2015.02.009

8. Penninx BW, Guralnik JM, Ferrucci L, Fried LP, Allen RH, Stabler SP. Vitamin B(12) deficiency and depression in physically disabled older women: epidemiologic evidence from the Women's Health and Aging Study. Am J Psychiatry 2000, 157 (5): 715-721. doi: https://doi.org/10.1176/appi.ajp.157.5.715

9. Mavrommati K, Sentissi O. Delirium as a result of vitamin B12 deficiency in a vegetarian female patient. Eur J Clin Nut 2013, 67 (9): 996-997. doi: https://doi.org/10.1038/ejcn.2013.128

10. Foster M, Samman S. Chapter Three - Vegetarian Diets Across the Lifecycle: Impact on Zinc Intake and Status. In: Henry J, editor. Advances in Food and Nutrition Research. 74: Academic Press; 2015. p. 93-131.

11. Lange KW. Omega-3 fatty acids and mental health. Global Health Journal 2020, 4 (1): 18-30. doi: https://doi.org/10.1016/j.glohj.2020.01.004 
12. Martínez-Cengotitabengoa M, González-Pinto A. Suplementos nutricionales en trastornos depresivos. Actas Esp Psiquiatr 2017, 45 (Supl. 1): 8-15.

13. Agarwal U, Mishra S, Xu J, Levin S, Gonzales J, Barnard ND. A Multicenter Randomized Controlled Trial of a Nutrition Intervention Program in a Multiethnic Adult Population in the Corporate Setting Reduces Depression and Anxiety and Improves Quality of Life: The GEICO Study. Am J Health Promot 2015, 29 (4): 245-254. doi: https://doi.org/10.4278/ajhp.130218-QUAN-72

14. Katcher HI, Ferdowsian HR, Hoover VJ, Cohen JL, Barnard ND. A worksite vegan nutrition program is well-accepted and improves health-related quality of life and work productivity. Ann Nutr Metab 2010, 56 (4): 245-252. doi: https://doi.org/10.1159/000288281

15. Medawar E, Enzenbach C, Roehr S, Villringer A, Riedel-Heller SG, Witte AV. Less Animal-Based Food, Better Weight Status: Associations of the Restriction of Animal-Based Product Intake with Body-Mass-Index, Depressive Symptoms and Personality in the General Population. Nutrients 2020, 12 (5): 1492. doi: https://doi.org/10.3390/nu12051492

16. Lei X-Y, Xiao L-M, Liu Y-N, Li Y-M. Prevalence of Depression among Chinese University Students: A Meta-Analysis. PLOS ONE 2016, 11 (4): e0153454. doi: https://doi.org/10.1371/journal.pone.0153454 